\title{
Optimization of a Tunnel Lining Reinforced with FRP
}

\author{
Ivana Laníková ${ }^{1, a^{*}}$, Petr Štěpánek ${ }^{1, b}$ and Jakub Venclovský ${ }^{1, c}$ \\ ${ }^{1}$ Brno University of Technology, Faculty of Civil Engineering, \\ Institute of Concrete and Masonry Structures,
}

Veveří 331/95, 60200 Brno,

\section{Czech Republic}

alanikova.i@fce.vutbr.cz, ${ }^{b}$ stepanek.p@fce.vutbr.cz, cvenclovsky.j@fce.vutbr.cz

Keywords: optimization of concrete structure, tunnel lining, FRP

\begin{abstract}
This contribution describes the formulation of DBSO (deterministic based structural optimization) design of a tunnel lining. The principle by which a solution may be obtained for a problem concerning the dimensional and reinforcement design optimization of a concrete structure is introduced. The target function is defined as the minimum cost of used materials. The range of allowable solutions from the mathematical viewpoint is defined by constraining conditions expressed by relations derived from:

- equations of equilibrium (the solution of optimization calculations for a structure with a Winkler foundation using the finite element method),

- the reliability conditions of a reinforced concrete structure

- and from the continuity of deformations.

The method (algorithm) for obtaining a serviceable reinforcement design (via the implementation of reinforcement types) from an optimal solution is described. The algorithm will be applied to the optimization of a tunnel lining design. FRP reinforcing bars will be used as reinforcement. The thickness of the tunnel lining and the areas of top and bottom reinforcement in cross-sections will be optimized. The obtained results will be compared with an optimized design created for the same tunnel structure but made from steel-reinforced concrete.
\end{abstract}

\section{Introduction}

Structural design constitutes a hugely complex web of architectural and engineering activities. All these activities are closely interconnected and work together to establish the quality of realized structures. It is a complicated endeavor which calls for the combination of the structural, aesthetic, operational, economic, energetic and environmental aspects of the construction field.

The aspects mentioned above are contradictory in nature: structural (choice of structural type, materials), aesthetic (exterior, environmental integration), operational (functionality, reliability and durability), economic (acquisition costs, maintenance costs, costs of modernization and regeneration) and environmental (the ecological impact associated with a structure's lifecycle). It is therefore necessary to carry out multi-criteria assessment and structural design optimization in order to ensure that expenditures made are invested effectively.

By definition of an optimization model it is necessary to define so called design variables. Design variables are a set of unknown parameters, owing to which a particular structure proposal is obtained (e.g. spans, spans number, shape and cross-sections dimensions, reinforcement amount, materials used etc.). They represent the design space and they have to be defined by their sets of all possibly acceptable values (discrete or continuous).

Each design of the solved structure, which is consistent with the prescribed restrictive conditions imposed on a structure under optimization, e.g. serviceability properties, the provisions of standards, reliability and structural requirements, etc., is termed a feasible solution. All of the vectors of design variables to which feasible solutions correspond constitute the set of feasible 
solutions. This set is generally very broad (by assumption the problem is meaningful, and therefore worth solving), and the goal is to choose (find) the design vector providing the best design in relation to the defined suitability criteria, i.e. so-called objective functions.

\section{Aim and Conditions for Execution}

The aim is to mathematically formulate and algorithmize the design optimization of a reinforced concrete structure in such a manner that it is safe and reliable, i.e. in order that it fulfills the following requirements:

- reliability conditions defined in the relevant standards with regard to ultimate limit states (ULS) and serviceability limit states (SLS),

- structural provisions according to the standards,

- other requirements and limits set by the user.

In order to be able to formulate and algorithmize an optimization problem, the task needs to be concretized and the area of design variables narrowed; simplifying conditions may also need to be introduced. In our case, it was assumed that:

- The geometry of the structure is strictly given by a static diagram (2D model); see Fig. 2 for a diagram of the problem solved.

- All material characteristics are strictly defined by the materials used.

- The optimized variables are the height, $h$, (or also width, $b$ ) of the individual rectangular cross sections and their reinforcements, which is given by the area of the reinforcement placed at the top and bottom surface $\left(A_{\mathrm{s} 1}\right.$ and $\left.A_{\mathrm{s} 2}\right)$, and the corresponding transverse reinforcement ( $A_{\mathrm{sr} 1}$ and $\left.A_{\mathrm{sr} 2}\right)$ : see Fig. 1.

- It isn't possible to express these requirements for all parts of the entire structure analytically. The structure needs to be discretized and the fulfillment of the loading capacity conditions in predefined cross sections is required.

- FEM analysis is used for the calculation of internal forces.

- Optimized values will be constant along the length of the element.

- ULS reliability conditions will be evaluated on a cross section stressed with a combination of bending moment and normal force. The effects of shear will not be dealt with.

- During the calculation of deformations in the structure for the evaluation of SLS reliability conditions, physical nonlinearity is considered (i.e. the weakening of the cross section due to the effect of cracks in concrete).

- The widths of cracks will not be assessed.

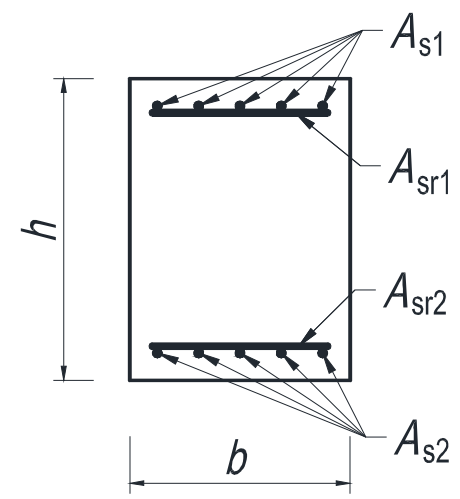

Figure 1: Optimized variables 


\section{Mathematical Formulation of the Optimization Task}

In general, the mathematical description of an optimization task which takes into account various design variables was published for example in [1,2].

Objective function. In the investigated task, the objective function is defined as the minimum structure cost, which corresponds to the price of the construction materials used, i.e. concrete and reinforcement:

$$
\min \left(C_{\mathrm{C}} V_{\mathrm{C}}+C_{\mathrm{S}} W_{\mathrm{S}}\right)
$$

where $C_{\mathrm{C}}$ and $C_{\mathrm{S}}$ are the cost of concrete per $\mathrm{m}^{3}$ and the cost of reinforcement per $\mathrm{kg}$, respectively, and $V_{\mathrm{C}}$ and $W_{\mathrm{S}}$ are the volume of concrete in $\mathrm{m}^{3}$ and the weight of reinforcement in $\mathrm{kg}$ used in the structure, respectively. It is possible to extend the objective function to include other aspects and evaluate the structure from the aspect of cost or the impact on the environment of the whole life cycle: see [3].

Constraining conditions. The requirements which the whole structure must fulfill are expressed with the aid of constraining conditions.

The ULS reliability conditions during the action of normal force and moment are expressed with the aid of checking the strain exerted on the reinforcement $\varepsilon_{\mathrm{sF}}$ and concrete $\varepsilon_{\mathrm{cF}}$ from the loading effects $N_{\mathrm{F}}$ and $M_{\mathrm{F}}$ in selected sections. They can be expressed using the inequalities:

$$
\begin{aligned}
& \varepsilon_{\mathrm{sE}} \leq \varepsilon_{\mathrm{sR}} \\
& \varepsilon_{\mathrm{cF}} \geq \varepsilon_{\mathrm{cR}}
\end{aligned}
$$

where $\varepsilon_{\mathrm{sR}}$ is the limit strain strength of the reinforcement and $\varepsilon_{\mathrm{cR}}$ is the limit strain in compression. The effect of loads (internal forces) $N_{\mathrm{F}}$ and $M_{\mathrm{F}}$ must correspond to the load, i.e. the balance of the structure must be maintained, which can be expressed with the aid of the finite element method (FEM) with the relationship:

$$
\boldsymbol{K}_{\mathrm{ULS}} \boldsymbol{u}_{\mathrm{ULS}}=\boldsymbol{F}_{\mathrm{ULS}},
$$

where $\boldsymbol{K}_{\mathrm{ULS}}$ is the stiffness matrix, $\mathbf{u}_{\mathrm{ULS}}$ is the vector of node deflections and $\boldsymbol{F}_{\mathrm{ULS}}$ is the vector of load corresponding to the combination of loading for the ULS.

The deformation of the structure (SLS), i.e. deflection or twisting $u$, is monitored in the nodes of the structure set by the user:

$$
u_{\min } \leq u_{\mathrm{SLS}} \leq u_{\max }
$$

where $u_{\max }$ or $u_{\min }$ is the limit value of the assessed deformation value. Again, the balance of the structure must be maintained and the following equation must be valid:

$$
\boldsymbol{K}_{\mathrm{SLS}} \boldsymbol{u}_{\mathrm{SLS}}=\boldsymbol{F}_{\mathrm{SLS}}
$$

where the values used correspond to the values in Eq. $2 \mathrm{c}$ but are determined for the $\boldsymbol{F}_{\mathrm{SLS}}$ loading values corresponding to the evaluated SLS. In the stiffness matrix $\boldsymbol{K}_{\mathrm{SLS}}$, the lowering of the stiffness of the elements due to the occurrence of cracks in the concrete is considered, as well as the influence of the tensile stiffening of concrete between the cracks.

The construction principles concerning the minimum acceptable area of the reinforcement in the individual layers, $A_{\mathrm{s}, \min }$, or the total maximum area of the reinforcement in the cross section, $A_{\mathrm{s}, \max }$, can be recorded as: 


$$
\begin{aligned}
& A_{\mathrm{s} 1}, A_{\mathrm{s} 2} \geq A_{\mathrm{s}, \min }, \\
& A_{\mathrm{s} 1}+A_{\mathrm{s} 2} \leq A_{\mathrm{s}, \text { max }} .
\end{aligned}
$$

Similarly, for transverse reinforcement:

$$
\begin{aligned}
& A_{\mathrm{sr} 1}, A_{\mathrm{sr} 2} \geq A_{\mathrm{sr}, \min }, \\
& A_{\mathrm{sr} i} \geq 0.25 A_{\mathrm{s}, i}, i=1,2 .
\end{aligned}
$$

The user can set other constraints, for example limit heights $\left(h_{\min }, h_{\max }\right)$ or the cross section width $\left(b_{\min }, b_{\max }\right)$ using the following inequalities:

$$
\begin{aligned}
& h_{\min } \leq h \leq h_{\max }, \\
& b_{\min } \leq b \leq b_{\max } .
\end{aligned}
$$

The optimization task itself is nonlinear (due to various relations between variables, e. $\mathrm{g}$. stiffness and height $h$, the nonlinear stress-strain diagram for concrete or steel), and uses the CONOPT solver [4] which is provided in GAMS modeling software [5] and is based on a reduced gradient algorithm. The task is composed of many situations where the usage of non-smooth functions is necessary (e.g. the approximation of stress-strain diagrams, the calculation of stiffness for cross-sections, including the impact of cracks in the concrete). However, the usage of such functions is precluded by the reduced gradient algorithm. Such situations are then resolved via Hermit's interpolation of these functions in their critical (non-smooth) parts. This is achieved in a different programming language (Delphi) with the aid of a DLL library. The solver then calls the function values and their derivations with the aid of so-called external equations implemented in an algorithm written in the GAMS language.

In order that the influence of the subsoil and stiffness matrix under equilibrium conditions can also be considered in equations, such equations also have to include the bending stiffness of the structure and the stiffness of the subsoil:

$$
\boldsymbol{K}_{\mathrm{j}}=\boldsymbol{K}_{\mathrm{b}, j}+\boldsymbol{K}_{\mathrm{s}, j} \text { for } j=\mathrm{ULS}, \mathrm{SLS},
$$

where $\boldsymbol{K}_{\mathrm{b}, j}$ is the matrix of bending stiffness and $\boldsymbol{K}_{\mathrm{s}, j}$ is the matrix of the soil-stiffness contribution. The interaction between the frame deflection and the reaction of the soil to this deflection is expressed by using Winkler's constant $k$ of the foundation $\left[\mathrm{N} / \mathrm{m}^{2}\right]$. In order to determine the internal forces acting on element outside the element node it is necessary to know the distribution of the soil-reaction field. For this reason, two-field interpolation was used to express stiffness $\boldsymbol{K}_{\mathrm{s}, j}$, i.e. one interpolation function for the displacements and rotations and a second for the linear approximation of the distributed soil-reaction field; see [6].

However, the Winkler soil model only represents a one-way binding between the soil and the mentioned frame. It is 'one-way' in the sense that when the frame deflects into the soil, it causes a reaction, while when the frame deflects away from the soil, the reaction is non-existent $(k=0$, which is equivalent to the original differential equation). This problem is solved by repeating the optimization cycle in such a way that at first the deflections are calculated and then, according to these deflections, the reactions / non-reactions with the soil are set for the respective frame elements. Another, more elegant way to solve this would be via the use of a sigmoid function. This would once again require the use of an alternative programming language and is being considered for the future. 


\section{Optimization of a Tunnel Lining}

Geometry. Our optimization problem concerns a utility tunnel, the original design geometry of which is shown in Fig. 2. The surrounding soil thus loads the tunnel structure (Fig. 3).

The mentioned width $b$ will be set to $b=1 \mathrm{~m}$. Thanks to the symmetry of the tunnel, calculations will only need to be performed for half of the frame. This half frame will be divided into 20 finite elements, $\mathrm{e}_{1}, \ldots, \mathrm{e}_{20}$, placed lengthwise along the tunnel lining, and 4 more elements, $\mathrm{e}_{21}, . ., \mathrm{e}_{24}$, placed on the bottom of the tunnel as shown in Fig. 2. The latter 4 elements are not subject to optimization and their thickness is a constant $600 \mathrm{~mm}$, as shown in Fig. 2.
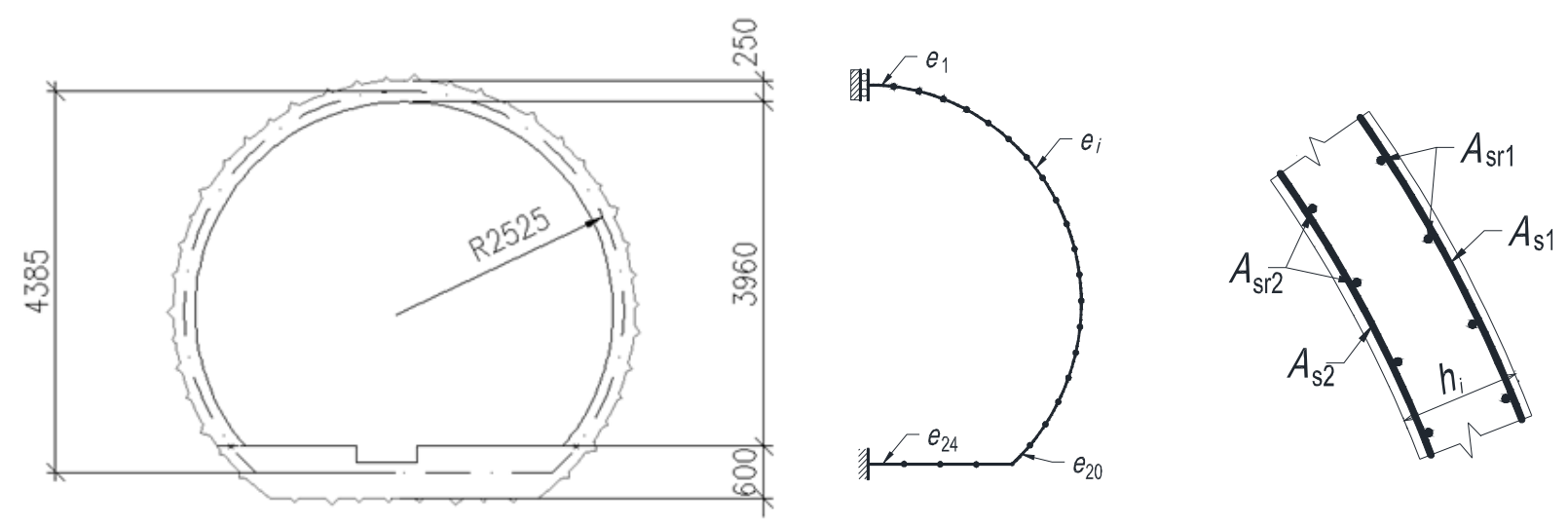

Figure 2: Cross-section of the solved structure; finite element discretization (beam elements)

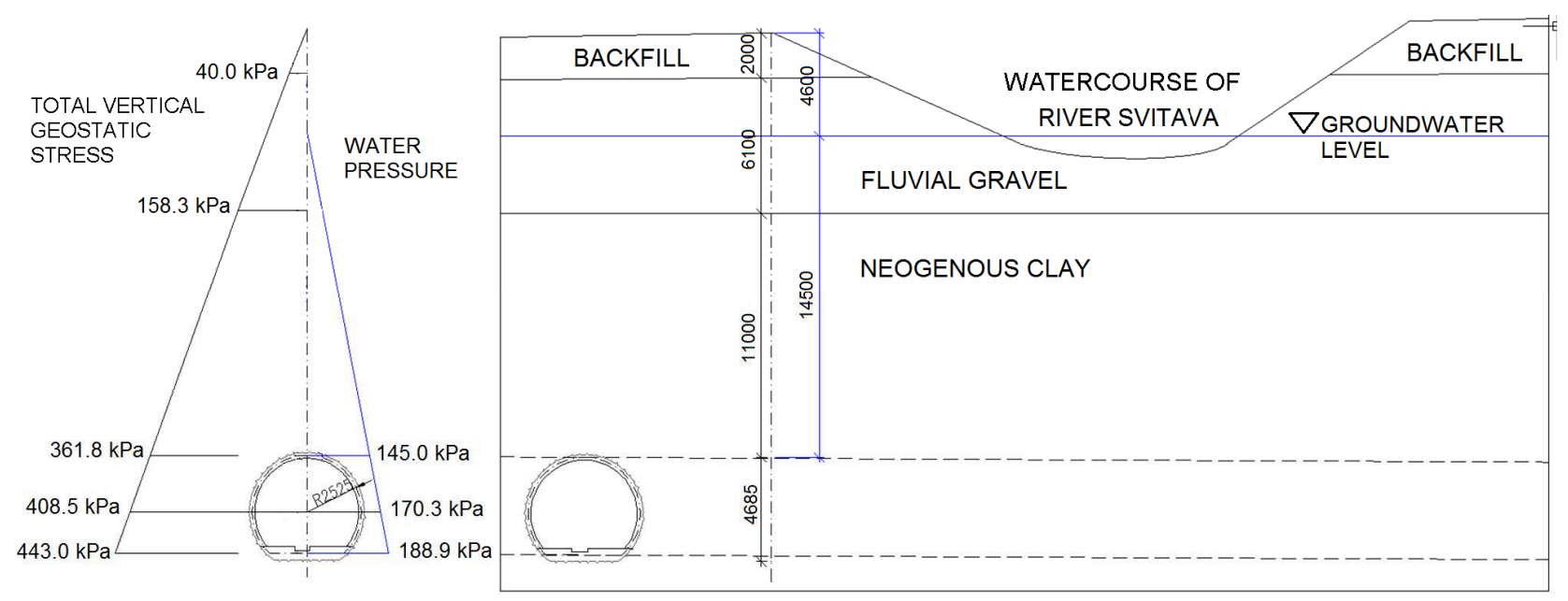

Figure 3: Layout of the collector structure

Target reliability. For the evaluation of reliability within ULS and SLS the semi-deterministic partial reliability factor method was used in accordance with European standards (EC) for fundamental persistent design situations and reliability class RC2. There is no possible to define the probability of failure and/or reliability index.

Load. The load on the tunnel is formed by pressure from the surrounding soil and underground water. The vertical component of this pressure can be seen in Fig. 3. The horizontal component of the soil pressure is calculated as the vertical component (soil pressure reduced by water pressure) multiplied by $K_{0}$. The horizontal component of the water pressure is the same as the vertical. Since the utility tunnel is constructed in soils with complicated geo-mechanical properties, it is necessary to consider two limit values for the $K_{0}$ coefficient. The first, $K_{0}=0.593$, is derived from Jaky's equation, while the second, $K_{0}=1.25$, is set manually according to the level of soil solidification 
(typical for neogenous clay in the Brno area). Because there are two $K_{0}$ limit values to express the vertical pressure, two different soil pressure load cases will be considered.

The vectors of load (the equilibrium conditions Eqs. $2 \mathrm{c}$ and $3 \mathrm{~b}$ ) are compiled for combinations of load cases (not for individual load cases) due to physical nonlinearity. It is predicted, that selfweight of frame, soil pressure and water pressure acts together; combinations without water pressure are not considered with regard to geological conditions.

The load in ULS is modified by means of partial reliability factors $\gamma_{\mathrm{F}}$ (favorable and unfavorable value). The values of the partial factors for self-weight and soil pressure meet the requirements of the Europeans standards (EC), while the partial factors for water pressure haves been adjusted to only one value $\gamma_{\mathrm{F}}=1$ instead of two values. The number of possible combinations (16) was reduced to 4 combinations in this case study (see Table 1) in which the partial factor of self-weight was always considered identical to the partial factor of soil pressure.

The equilibrium conditions of the SLS are solved for two load combinations (because of the two values of the coefficient $K_{0}$ ) for the partial factors $\gamma_{\mathrm{F}}=1$.

Table 1: Partial factor $\gamma_{\mathrm{F}}$ for combinations of load cases (ULS)

\begin{tabular}{|l|c|c|c|c|}
\hline Combination of load cases & CL1 & CL2 & CL3 & CL4 \\
\hline Coefficient $K_{0}$ & \multicolumn{2}{|c|}{0.593} & \multicolumn{2}{|c|}{1.25} \\
\hline Frame weight & 1 & 1.35 & 1 & 1.35 \\
\hline Soil pressure & 1 & 1.35 & 1 & 1.35 \\
\hline Hydrostatic pressure & 1 & 1 & 1 & 1 \\
\hline
\end{tabular}

Great compressive force and bending moments arise in the lining of the tunnel due to the strain exerted upon it. In order to take inaccuracies in the geometry of the structure into consideration, moments were adjusted by $0.020 \mathrm{~m}$ to the dangerous side, as stipulated in EC standards for ULS, in order to include possible imperfections in the position of the effect of the normal force with regard to the cross-section center of gravity.

Used materials. As regards concrete, shotcrete corresponding to concrete with a strength class of $\mathrm{C} 25 / 30$ will be used. The cost will be $C_{\mathrm{C}}=1058 \mathrm{USD} / \mathrm{m}^{3}$ for the inner layers and $C_{\mathrm{C}}=1422 \mathrm{USD} /$ $\mathrm{m}^{3}$ for the outer layers (the outer layer is considered to be $10 \mathrm{~mm}$ thick).

In this study, two different reinforcement variants are considered:

- B500B steel (cost $C_{\mathrm{S}}=1.57 \mathrm{USD} / \mathrm{kg}$ );

- reinforcement fabricated from alkali resistant (AR) glass fiber reinforced polymer (GFRP).

The idealized strain-stress diagram for the GFRP reinforcement under tensile strain is linear up until failure occurs. The short-term characteristic tensile strength $(5 \%$ quantile according to EC standards) is $609 \mathrm{MPa}$ and the corresponding characteristic limit strain value is $1.73 \%$. These values for FRP with AR glass fibers have to be reduced according to the expected design life. In this case, the long-term strength was considered as $40 \%$ of short-term strength. The modulus of elasticity is $36 \mathrm{GPa}$ (mean value). The effect of reinforcement in compression was not considered when calculating the load capacity of the cross section. The unit weight of the reinforcement is relatively low: $21 \mathrm{kN} / \mathrm{m}^{3}$. More information on the characteristics of the tested reinforcement and its application is in [7].

For this reinforcement, the cost depends on the size of the profile as shown in Table 2. In the optimization algorithm, the sought area, $A_{\mathrm{s}}$, represents the area of the reinforcement per $1 \mathrm{~m}$ of width of the lining. As a result, the cost of one kilogram of reinforcement with an area of $A_{\mathrm{S}}$ was calculated from the price of a single bar, using the assumption that individual bars are placed in the cross section every $150 \mathrm{~mm}$.

The cost of one kilogram of reinforcement depends on the area of the reinforcement, $A_{\mathrm{s}}$ (see Table 2), and it was approximated via a suitable function for the purpose of the required calculations (see Fig. 4). 
Table 2: Price of GRRP reinforcement

\begin{tabular}{|c|c|c|c|}
\hline \multirow{2}{*}{ Diameter of bar } & \multirow{2}{*}{ Cost of bar (1m length) } & \multicolumn{2}{|c|}{ Area of reinforcement and price per 1 m of width } \\
\cline { 2 - 4 } & {$[\mathrm{USD} / \mathrm{m}]$} & $\mathrm{A}_{\mathrm{s}}$ & Cost $\mathrm{C}_{\mathrm{S}}$ \\
\hline$[\mathrm{mm}]$ & 1.57 & 130 & $\mathrm{USD} / \mathrm{kg}$ \\
\hline 5 & 1.65 & 188 & 38.08 \\
\hline 6 & 1.78 & 335 & 27.84 \\
\hline 8 & 1.98 & 523 & 16.83 \\
\hline 10 & 2.23 & 754 & 12.02 \\
\hline 12 & 2.52 & 1026 & 9.39 \\
\hline 14 & 2.85 & 1340 & 7.80 \\
\hline 16 & 3.51 & 1696 & 6.75 \\
\hline 18 & 4.75 & 2094 & 6.57 \\
\hline 20 & 5.17 & 2534 & 7.20 \\
\hline 22 & & & 6.47 \\
\hline
\end{tabular}

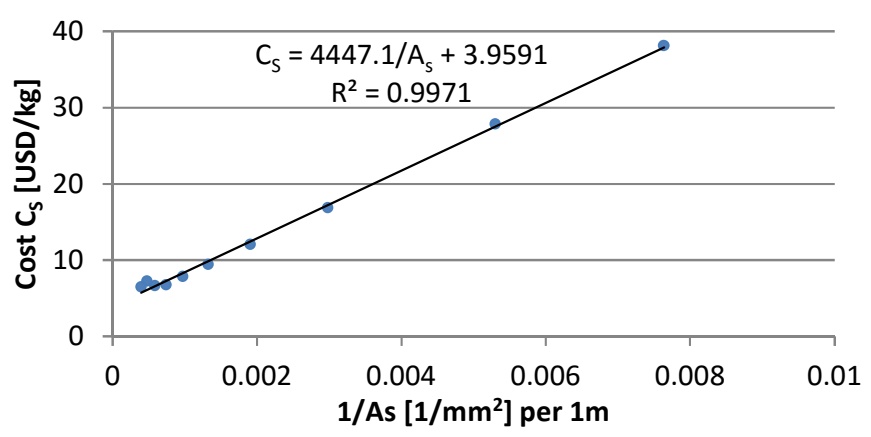

Figure 4: Approximation of the price function

\section{Case Study}

A case study was carried out for several tunnel lining optimization variants. Its aim was to obtain an idea about the change in the height of the cross section and the areas of the reinforcement along the perimeter of the tunnel lining. Optimized values $x$ (i.e. the height of the cross section $h$, the reinforcement areas $A_{\mathrm{s} 1}, A_{\mathrm{s} 2}$ ) for each element were either left independent or were bound by the following constraining condition:

$$
x\left(e_{1}\right)=x\left(e_{2}\right)=\ldots=x\left(e_{20}\right),
$$

i.e. so that they were constant along the perimeter of the lining. The analyzed variants are described in Table 3.

Table 3: Analyzed variants

\begin{tabular}{|l|l|l|l|l|l|l|}
\hline \multicolumn{4}{|l|}{ Steel reinforcement } & \multicolumn{4}{l|}{ GFRP reinforcement } \\
\hline Variants & CS-S1 & CS-S2 & CS-S3 & CS-G1 & CS-G2 & CS-G3 \\
\hline$h$ & independent & constant & constant & independent & constant & constant \\
\hline$A_{\text {si }}{ }^{1)}$ & independent & independent & constant & independent & independent & constant \\
\hline
\end{tabular}

${ }^{1)}$ Independently for both layers of the reinforcement.

The decisive criterion was the fulfillment of ULS reliability conditions in cross sections which were always placed at the beginning and end of each finite element and SLS constraining condition for the limitation of the vertical deflection of the top node of the lining $( \pm 35 \mathrm{~mm})$. The elements at the bottom of the tunnel were not subjected to optimization and are not included in the objective function (1). The results of the analyzed variants are compared in Table 4, while the values of variables which are not constant along the perimeter of the lining are shown in Fig 5 and 6 . The 
costs are stated for the whole lining for a width of $1 \mathrm{~m}$ (i.e. the objective values of the optimization are multiplied by 2).

Variants CS-S1 and CS-G1 (variable values for the height and areas of the reinforcement along the perimeter of the lining) represent the lowest possible value obtained for the objective function, while variants CS-S3 and CS-G3 (constant values for the height and areas of the reinforcement along the perimeter of the lining) attain the highest possible objective function values.

Table 4: Comparison of results

\begin{tabular}{|c|c|c|c|c|c|c|c|}
\hline \multirow{2}{*}{$\begin{array}{l}\text { Reinforcement } \\
\text { Variant }\end{array}$} & & \multicolumn{3}{|l|}{ Steel } & \multicolumn{3}{|l|}{ GFRP } \\
\hline & & CS-S1 & CS-S2 & CS-S3 & CS-G1 & CS-G2 & CS-G3 \\
\hline \multicolumn{8}{|c|}{ Optimized variables } \\
\hline$H$ & {$[\mathrm{~mm}]$} & 1) & 175.5 & 191.3 & 1) & 300.8 & 338.4 \\
\hline$A_{\mathrm{s} 1}$ & {$\left[\mathrm{~mm}^{2}\right]$} & 1) & 2) & 2057 & 1) & 2) & 6703 \\
\hline$A_{\mathrm{s} 2}$ & {$\left[\mathrm{~mm}^{2}\right]$} & 1) & 2) & 3644 & 1) & 2) & 750 \\
\hline \multicolumn{8}{|l|}{ Cost } \\
\hline Concrete & [USD] & 2281 & 2379 & 2582 & 2967 & 3984 & 4466 \\
\hline reinforcement & [USD] & 518 & 480 & 1063 & 865 & 723 & 1405 \\
\hline Total & [USD] & 2799 & 2859 & 3646 & 3832 & 4707 & 5871 \\
\hline \multicolumn{8}{|c|}{ Vertical deflection of node in top of lining } \\
\hline CL1 & {$[\mathrm{mm}]$} & -20.6 & -20.1 & -17.6 & -33.6 & -28.3 & -25.1 \\
\hline CL2 & {$[\mathrm{mm}]$} & 4.5 & 4.5 & 2.7 & -4.6 & -12.2 & -8.6 \\
\hline
\end{tabular}

${ }^{1)}$ See Fig. 5.

2) See Fig. 6.
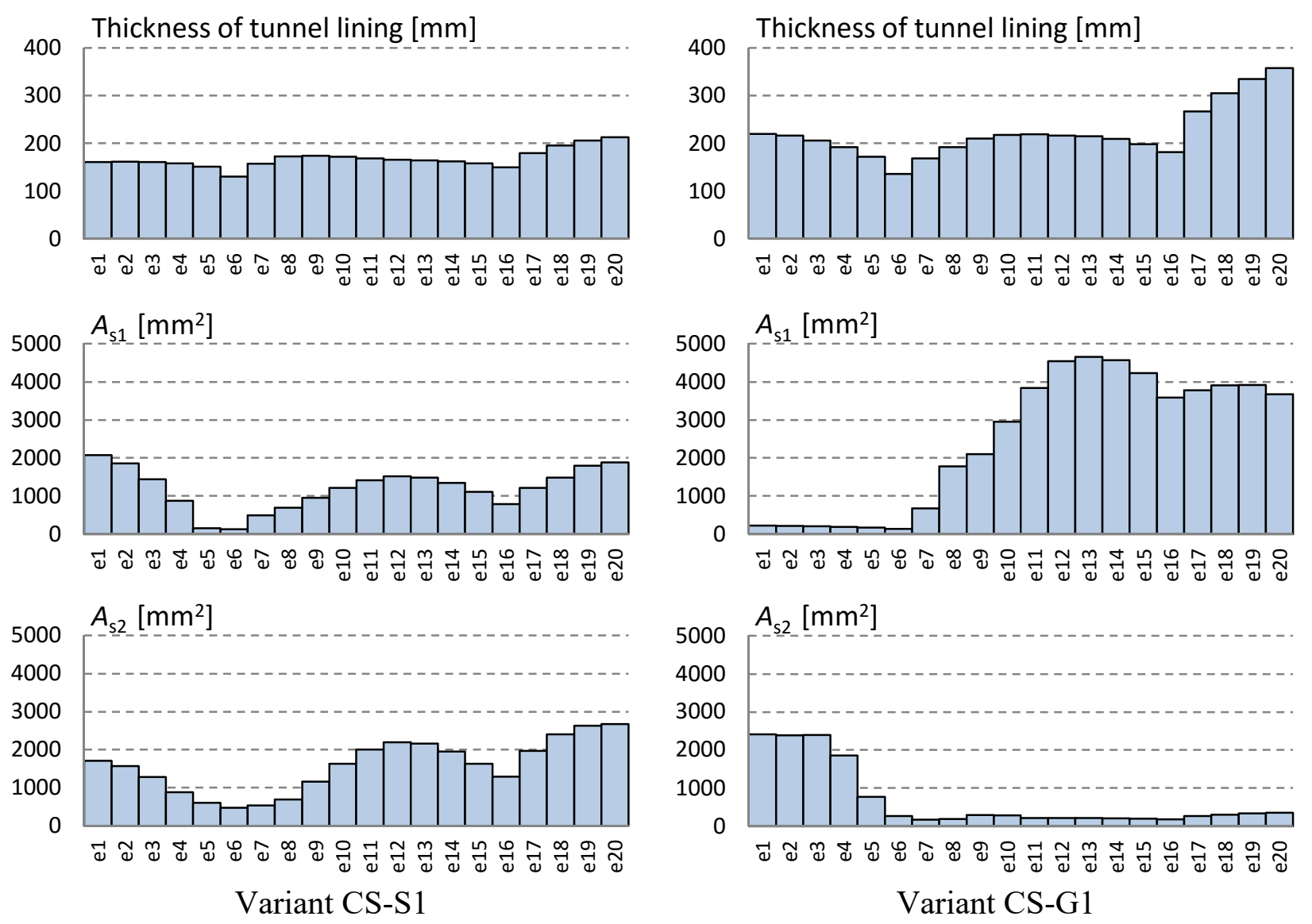

Fig. 5: Thickness of tunnel lining and reinforcement areas $A_{s 1}$ (backside of tunnel lining) and $A_{s 2}$ (face side of tunnel lining) for variants CS-S1 and CS-G1. 

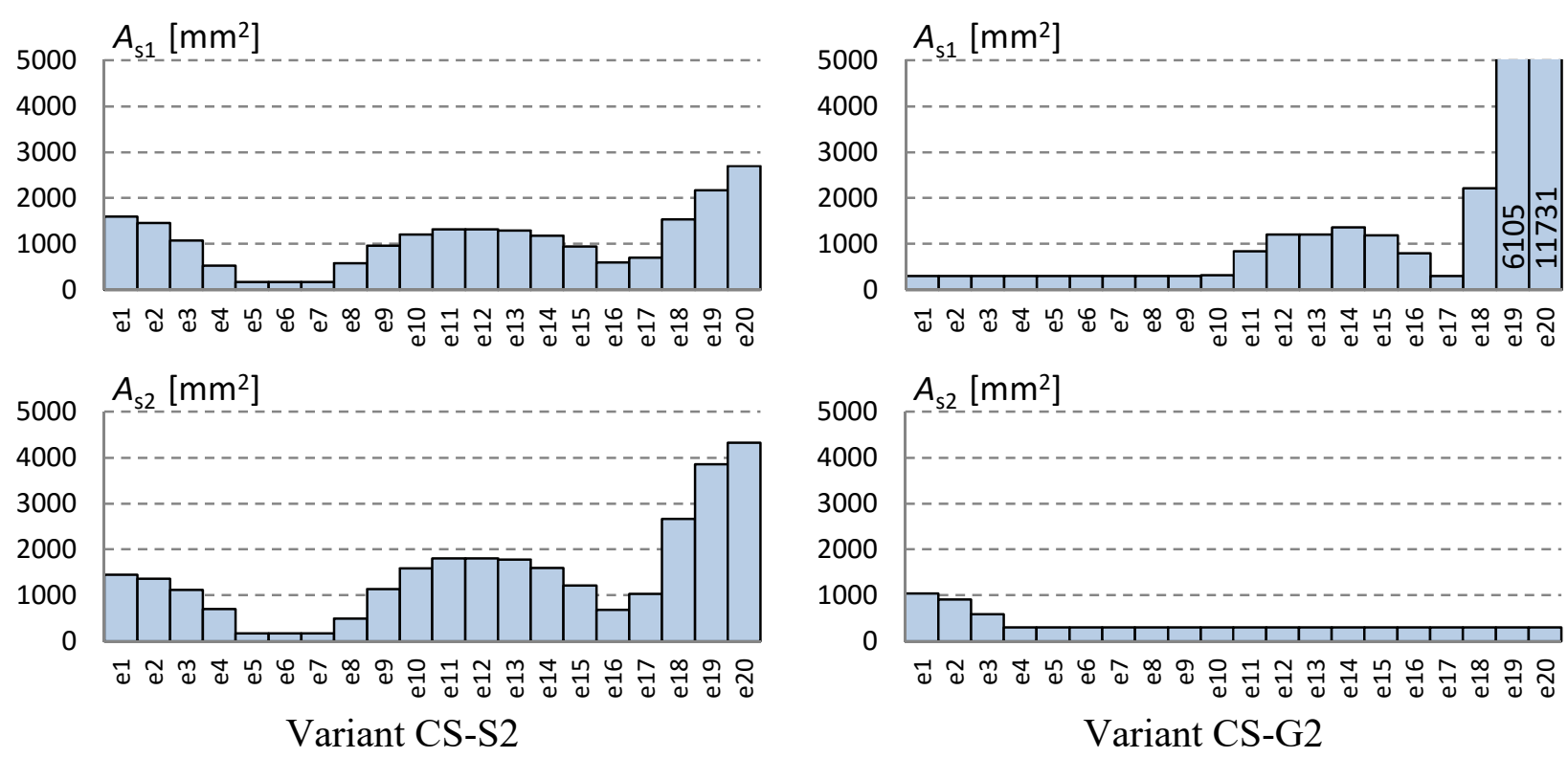

Fig. 6: Reinforcement areas $\mathrm{A}_{\mathrm{s} 1}$ and $\mathrm{A}_{\mathrm{s} 2}$ for variants CS-S2 and CS-G2, $h=$ constant.

For the lining variants with GFRP reinforcement, larger reinforcement areas and greater cross section heights were found to be necessary (resulting from the calculations) than for the lining variants with concrete reinforcement. Due to the influence of the low elasticity modulus and not calculating the FRP reinforcement in compression (it was allowed no effect), the stiffness of the structure is lower, as is obvious from the vertical shifts of the node in the middle of the lining: see Table 4. In addition, the values of the necessary areas of both layers of reinforcement along the perimeter of the tunnel are different in both materials, the reason once again being the use of only tensile reinforcement for the transmission of forces in the GFRP reinforced cross section.

With regards to these facts and the higher cost of GFRP reinforcement, the price of the lining is higher.

\section{Practical Proposal}

The solution of the previously introduced problem will obviously provide different values of $h$, $A_{\mathrm{s} 1}$ and $A_{\mathrm{s} 2}$ for each of the different elements $e$ that form the optimized frame (unless they are bound to each other by default). Since the tunnel lining will be constructed using shotcrete, the different $h$ values pose no problem. This does not apply for $A_{\mathrm{s} 1}$ and $A_{\mathrm{s} 2}$, though. The placement of so many different steel profiles would be impractical and probably not worth the effort. Therefore, the next step will be to introduce reinforcement types, which is equivalent to dividing the set $E$ of elements $e$ that form the frame into a prescribed number $n$ of subsets $E_{1 i}, i=1, \ldots, n$, where in each subset the value of $A_{s 1}$ must be the same (these subsets must contain consequent elements):

$$
\begin{aligned}
& A_{\mathrm{s} 1}\left(e_{j}\right)=A_{\mathrm{s} 1}\left(e_{k}\right) \forall e_{j}, e_{k} \in E_{1 i}, i=1, \ldots, n, \\
& \bigcup_{i=1}^{n} E_{1 i}=E .
\end{aligned}
$$

This occurs analogically for $A_{\mathrm{s} 2}$ and subsets $E_{2 i}, i=1, \ldots, n$.

Every single reinforcement bar must be properly anchored. This means that the actual bar length is composed of its effective length and its anchorage length. The anchorage length will be added to each subset. The anchorage length depends on the reinforcement profile and bond resistance.

For the solution to be viable, some assumptions must be made:

- individual bars are placed at a distance of $150 \mathrm{~mm}$ from each other, enabling the reinforcement profile to be derived from areas $A_{\mathrm{s} 1}$ and $A_{\mathrm{s} 2}$, 
- the anchorage length is equal to reinforcement profile multiplied by 30 for steel and 40 for FRP reinforcement (bonding condition),

- the anchorage length is added twice to each reinforcement bar (to the beginning and end of the bar), except for the first reinforcement type, which includes element $e_{1}$ to which the anchorage length is added only once (as it is next to the axis of symmetry of the tunnel).

The amount of material used for anchoring is then added to the overall weight of reinforcement in the objective function.

Two variants were investigated. With the first one (D-S1 for steel and D-G1 for FRP reinforcement), the height of the lining was the expected variable, which is possible when shotcrete is used. The reinforcement in each of the layers was divided into two reinforcement types $(n=2)$ on the basis of results obtained for variants CS-S1 and CS-G1: see Fig. 5. The division is obvious from the results in Fig. 7.
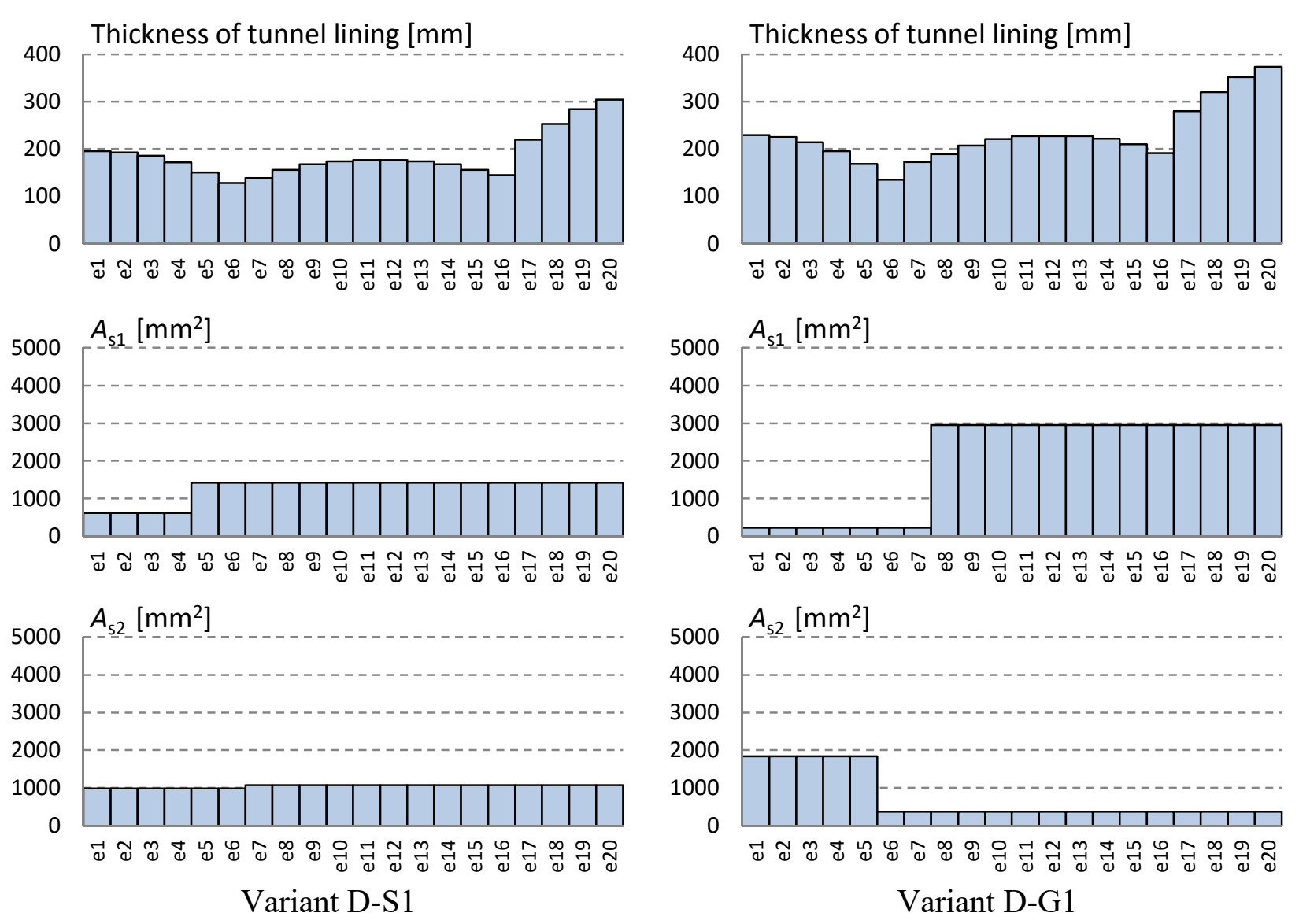

Fig. 7: Thickness of tunnel lining and reinforcement areas $A_{s 1}$ and $A_{s 2}$ for variants D-S1and D-G1

For comparison, other variants (D-S2 for steel and D-G2 for GFRP reinforcement) were also calculated but with the additional set condition of having the same lining thickness, i.e. Eq. 6. The reinforcement types were again determined according to the results in Fig. 7, which depicts the reinforcement areas of variants CS-S2 and CS-G2 with a constant lining thickness. For steel, the division into reinforcement types remained the same as in the case of the previous variant, and another division was selected for the GFRP reinforcement. The division can be clearly seen in Fig. 8.

Table 5 contains a complete comparison of the results. The condition of a constant lining height increased the cost of the structure by $27 \%$ if steel reinforcement was used and $44 \%$ for GFRP reinforcement. 

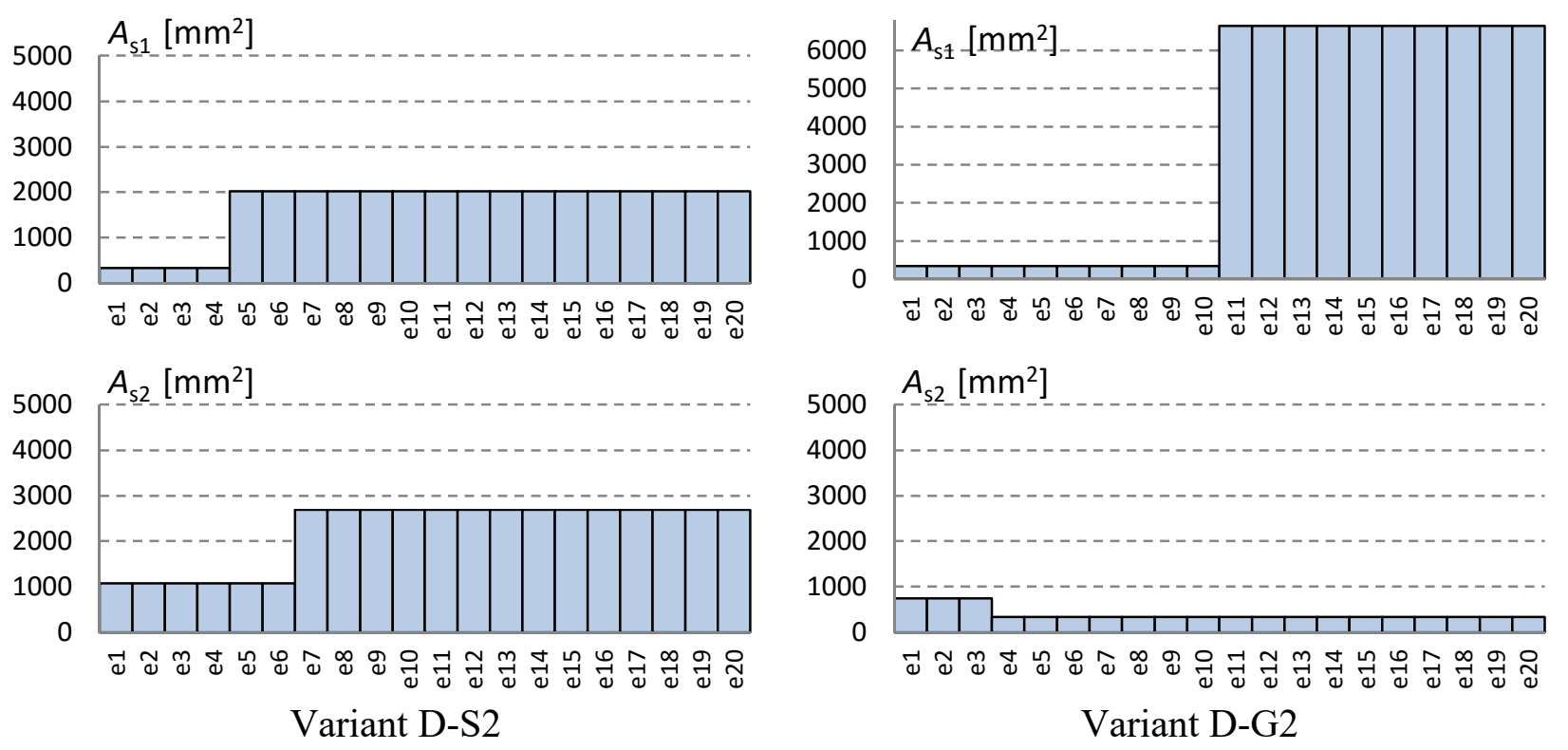

Fig. 8: Reinforcement areas $\mathrm{A}_{\mathrm{s} 1}$ and $\mathrm{A}_{\mathrm{s} 2}$ for variants D-S2and D-G2, $h=$ constant.

Table 5: Comparison of results

\begin{tabular}{|c|c|c|c|c|c|}
\hline \multirow{2}{*}{\multicolumn{2}{|c|}{$\begin{array}{l}\text { Reinforcement } \\
\text { Variant } \\
\end{array}$}} & \multicolumn{2}{|l|}{ Steel } & \multicolumn{2}{|l|}{ GFRP } \\
\hline & & D-S1 & $\mathrm{D}-\mathrm{S} 2$ & D-G1 & $\mathrm{D}-\mathrm{G} 2$ \\
\hline \multicolumn{6}{|c|}{ Optimized variables } \\
\hline$h$ & {$[\mathrm{~mm}]$} & 1) & 216.1 & 1) & 339.0 \\
\hline \multirow{2}{*}{$A_{\mathrm{s} 1}$} & \multirow{2}{*}[\mathrm{mm}^{2}]{} & $620^{1)}$ & $339^{2)}$ & $230^{1)}$ & $339^{2)}$ \\
\hline & & $1417^{1)}$ & $2022^{2)}$ & $2952^{1)}$ & $6646^{2)}$ \\
\hline \multirow{2}{*}{$A_{\mathrm{s} 2}$} & \multirow{2}{*}[\mathrm{mm}^{2}]{} & $988^{1)}$ & $1070^{2)}$ & $1835^{1)}$ & $747^{2)}$ \\
\hline & & $1070^{1)}$ & $2685^{2)}$ & $374^{1)}$ & $339^{2)}$ \\
\hline \multicolumn{6}{|l|}{ Cost } \\
\hline concrete & [USD] & 2511 & 2900 & 3069 & 4474 \\
\hline reinforcement & [USD] & 503 & 891 & 933 & 1305 \\
\hline total & [USD] & 3014 & 3791 & 4003 & 5779 \\
\hline \multicolumn{6}{|c|}{ Vertical deflection of node in top of lining } \\
\hline CL1 & {$[\mathrm{mm}]$} & $\begin{array}{r}-19.1 \\
\end{array}$ & -11.6 & -33.2 & -25.3 \\
\hline CL2 & {$[\mathrm{mm}]$} & 3.4 & 2.3 & -5.1 & -8.6 \\
\hline
\end{tabular}

${ }^{1)}$ Placement along the perimeter of the lining, see Fig. 7.

${ }^{2)}$ Placement along the perimeter of the lining, see Fig. 8.

\section{Conclusion}

It can be concluded from the comparison of variants with concrete reinforcement and those with GFRP reinforcement that the production cost of $1 \mathrm{~m}$ of tunnel lining reinforced with GFRP reinforcement is higher in all cases: see Fig. 9. For a variable lining thickness the increase in price is approximately $33 \%$, while for variants with a constant lining thickness it is almost $52 \%$.

In the case of using FRP reinforcement the strain of the outer layer of concrete decided on carrying capacity of cross-section subjected to normal force and bending moment (ULS), namely in all cross-sections under consideration. This means that the tensile reinforcement is not fully utilized and the stress is less than the ultimate long-term strength.

However, concrete elements exposed to the effects of an aggressive environment in which corrosion of reinforcement occurs due to the influence of the development and propagation of cracks are a promising area of application for composite reinforcement (FRP). At present, the 
advantages of using these modern reinforcement materials are well known and it is thus clear that the use of non-corroding and very resistant (from the aspect of environmental stress) reinforcement leads to the maximization of the lifespan of structural elements. The design lifespan of an element with correctly-designed non-metal reinforcement is theoretically unlimited; practically speaking, it is determined by the manufacturing quality of the element and the lifespan of the concrete parts of the cross section.
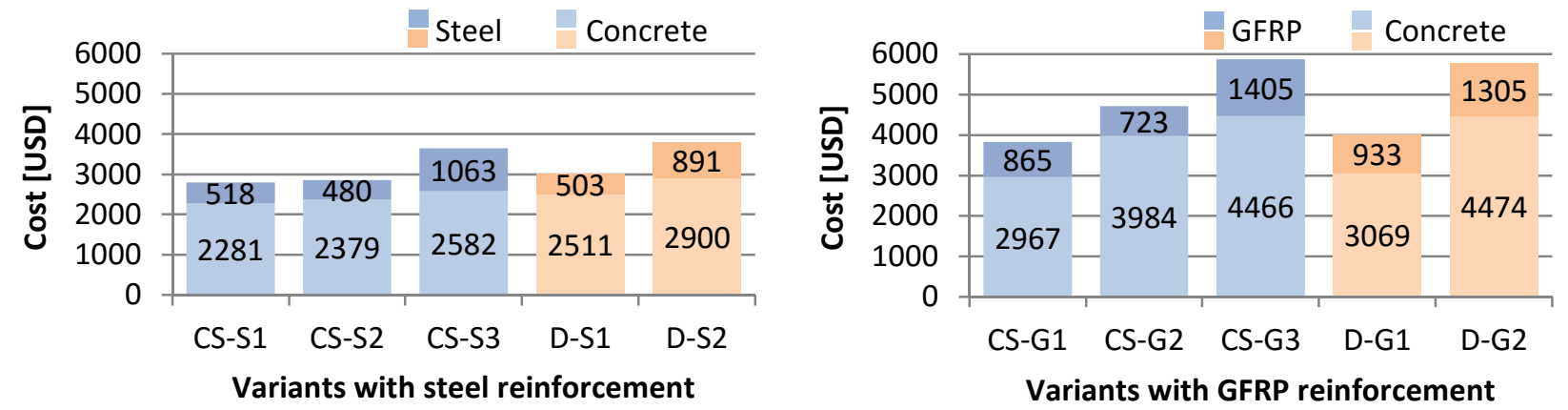

Fig. 9: Comparison of prices.

However, if an investor considers a structural element reinforced with composite reinforcement with regard to the whole life cycle of a structure for which the costs of maintenance and restoration work have to be included in the total price, the use of composite reinforcement appears to be completely competitive.

\section{Acknowledgement}

This paper has been produced under project No. LO1408 "AdMaS UP - Advanced Materials, Structures and Technologies", supported by the Ministry of Education, Youth and Sports under "National Sustainability Programme I".

\section{References}

[1] Carmichael, D.J., Structural modelling and optimization, Chichester, England: Ellis Horwood, 1981.

[2] Bhatti M.A., Practical Optimization Methods: With Mathematica ${ }^{\circledR}$ Applications, first ed., Springer-Verlag, New York, 2000.

[3] Laníková, I., Štěpánek, P., Šimůnek, P.: Optimized Design of Concrete Structures Considering Environmental Aspects, Advances in Structural Engineering, 2014 (vol. 17), No. 4, pp: 495-511.

[4] Drud, A. S., CONOPT: A System for Large Scale Nonlinear Optimization. ARKI Consulting and Development A/S, Bagsvaerd, Denmark,1996.

[5] General Algebraic Modeling System (GAMS), http://www.gams.com/

[6] Jagodnik, V., Jelenić, G., Arbanas, Ž.: The mixed-type approach to finite-element analysis of geometrically linear beams resting on linear and non-linear Winkler soil models (2014) Numerical Methods in Geotechnical Engineering - Proceedings of the 8th European Conference on Numerical Methods in Geotechnical Engineering, NUMGE 2014, 1, pp. 241-245.

[7] Ďurech, D., Štěpánek, P., Horák, D.: Renovation of a bridge using GFRP reinforced concrete slab. Proceedings: CESB 2010 Prague - Central Europe towards Sustainable Building 'From Theory to Practice'. pp. 1-13. 